\title{
Oferta de leña en bosques nativos altoandinos de la Cordillera del Vilcanota-Cusco
}

\author{
Firewood offer in high Andean native forests of the Vilcanota Mountain range-Cusco
}

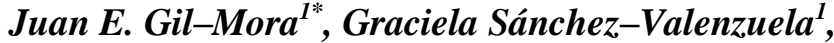 \\ Jesús A. Baca-Flores ${ }^{2}$,Erick F. Gil-Rodríguez ${ }^{I}$ \\ ${ }^{1}$ Escuela Profesional de Biología, Facultad de Ciencias, Universidad Nacional de San Antonio Abad del Cusco-Perú, \\ Av. La Cultura 733, Cusco, Perú \\ ${ }_{2}^{2}$ Escuela Profesional de Física. Facultad de Ciencias. Universidad Nacional de San Antonio Abad del Cusco-Perú. \\ *Autor corresponsal: Juan E. Gil-Mora, mundoandino2005@yahoo.es
}

\begin{abstract}
RESUMEN
Las especies leñosas tienen importancia en la economía de las comunidades campesinas altoandinas del Cusco, desde hace décadas se observa la creciente escasez de leña para uso doméstico. El objetivo del trabajo fue evaluar la oferta de leña que brindan los bosques nativos en los altos andes en las dos zonas de trabajo. El estudio se desarrolló en bosques nativos ubicados por encima de los $3200 \mathrm{~m}$ de altitud en la cordillera del Vilcanota, en las microcuencas de los ríos Patacancha y Málaga, (distrito de Ollantaytambo, Provincia de Urubamba) y los ríos Lares (distrito de Lares) y Warán (distrito de Calca), ambos en la provincia de Calca; la información se obtuvo mediante trabajos de campo e investigación participativa utilizando transectos en banda y parcelas cuadradas; para el análisis se utilizó la estadística descriptiva. Se evaluaron 829 hectáreas de bosques pertenecientes a 20 bosquetes clasificados como bosques mixtos con 510 ha; 159 ha de bosques de Polylepis; 135 ha, de matorral espinoso y 25 ha de rodales. La densidad ecológica determinada fue de 580,75 árboles/ha y la oferta de leña total calculada es de 215,70 t/ha; en las comunidades ubicadas entre 3200 y $3500 \mathrm{~m}$ de altitud, los bosques mixtos, matorrales espinosos y asociaciones interespecíficas, son los que proporcionan madera y leña en cantidades aún suficientes para el requerimiento de las comunidades; en cambio, en los bosques localizados por encima de los $3500 \mathrm{~m}$ de altitud, las especies de Polylepis son las que proveen las mayores ofertas de leña.
\end{abstract}

Palabras clave: Bosque nativo, densidad ecológica, madera, matorral, servicios ecosistémicos.

\begin{abstract}
Woody species are important in the economy of the high Andean peasant communities of Cusco and, for decades, the growing shortage of firewood for domestic use has been observed. The objective of the research is to evaluate the supply of firewood provided by native forests in the high Andes, as well as the demand of the high Andean communities studied. The study was developed in native forests located above 3200 m.a.s.l in the Vilcanota mountain range, in the micro-basins of the rivers: Patacancha and Málaga (Ollantaytambo district, Urubamba province) and Lares river (Lares district) and Warán river (Calca district), both of them in Calca province); the information was obtained through field work and participatory research using band transects and square plots; descriptive statistics were used for the analysis. 829 hectares of forests belonging to 20 forests classified as mixed forests with 510 ha were evaluated; 159 ha of Polylepis forests; 135 ha of thorny scrub and 25 ha of stands. The ecological density determined was 580.75 trees/ha and the total supply of firewood calculated is $215.70 \mathrm{t} / \mathrm{ha}$; in the communities located between 3200 and $3500 \mathrm{~m}$ of altitude, the mixed forests, thorn bushes and interspecific associations are those that provide wood and firewood in quantities that are still sufficient for the communities' requirements; on the other hand, in communities located above 3500 m.a.s.l. Polylepis species are those that provide the greatest supply of firewood.
\end{abstract}

Key words: Ecosystem services, ecological density, native forest, scrub, wood.

\section{INTRODUCCIÓN}

Los bosques naturales en los andes son recursos renovables que pueden dar una producción permanente de servicios ecosistémicos (FAO, 1983; Bermejo \& Passeti, 1985). El bosque andino es el remanente forestal y, se halla en riesgo de extinguirse por la agricultura y ganadería (Loján, 1992). MINAGRI (2012), concluye que el uso como material de construcción o fuente de energía, agota la biomasa vegetal. Las comunidades campesinas de los altos andes, ubicadas en la cordillera del Vilcanota usan productos del bosque como fuente de energía (leña) proveniente de bosques relictos dominados por Escallonia, Polylepis, Gynoxys, Buddleja, Alnus, Citharexylum, Myrcianthes, Weinmannia, Myrica, Duranta, etc.

Los Andes constituyen una de las regiones del planeta donde la escasez de leña es aguda (FAO, 1981, 1983); siendo las regiones altiplánica y altoandina las más afectadas, en estos espacios la leña se ha utilizado a tal punto que la población ya no está en condiciones de satisfacer sus necesidades mínimas (Ocaña, 1994; Gil, 2002).

El consumo de leña y carbón vegetal por la población rural ha variado debido al cambio de los hábitos energéticos de la población que usan ahora más gas o electricidad para la preparación de alimentos (MEM, 2010). Esta situación de cambio energético no es reflejada en los anuarios forestales cuyas cifras de la producción de leña y carbón vegetal para consumo es el mismo año tras año. Los datos oficiales del MINAGRI para 2012, indican que el consumo de leña y carbón vegetal representa la conversión del $90 \%$ del volumen de madera extraída del país a energía. De otro lado, el Balance Nacional de Energía, documento preparado por el Ministerio de Energía y Minas que permite conocer la estructura del sector energético nacional, aun cuando no especifica la fuente proveniente del consumo de leña y los cambios respecto al año anterior y determinar el impacto en el ambiente (MEM, 2010). Geist \& Lambin, 2001, sostienen que el uso intensivo del suelo, ha producido el empobrecimiento y erosión; a pesar de ello, el campesino sigue cultivando en las laderas y en suelos marginales; Ehrhardt-Martinez, 1998, concluyen que la deforestación tiene consecuencias sociales.

La presente investigación evalúa la oferta de leña que brindan los bosques nativos en los altos andes; el estudio evidencia un análisis sobre los bosques remanentes en los altos andes utilizados como combustible por comunidades 


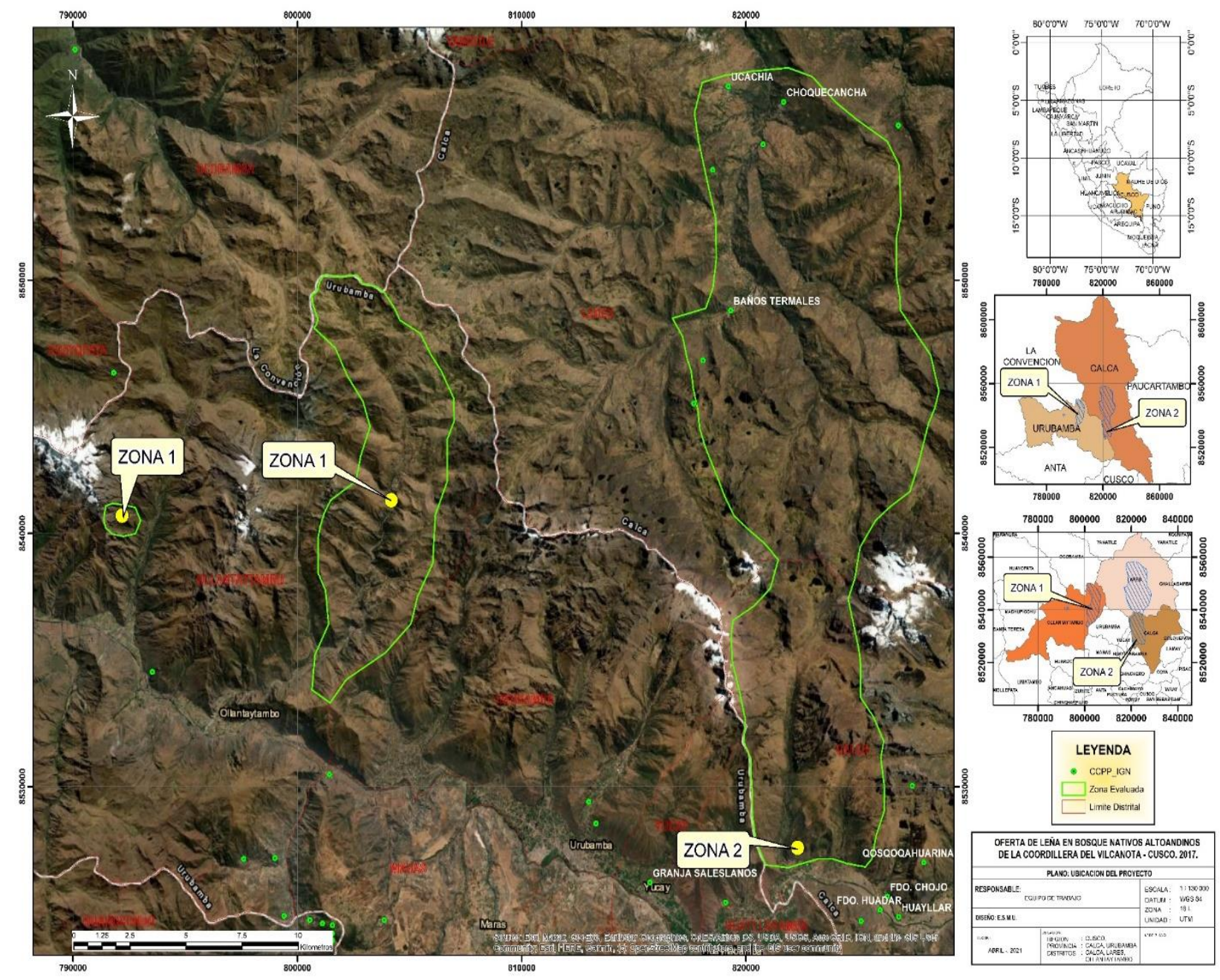

Figura 1. Mapa de ubicación y ámbito de estudio

campesinas, además, se identificaron problemas del uso de las especies utilizadas como leña. Para la determinación de la oferta de leña, se utilizó la definición de leña como la materia leñosa y celulósica de troncos y ramas de árboles y arbustos utilizados como combustible para cocinar y para calefacción (FAO, 1983).

La demanda de leña por las comunidades campesinas estudiadas y la oferta de leña y otros servicios ambientales brindados por los bosques, deben ser conocidos a fin de proponer acciones de protección, manejo y conservación; además de conocer las especies más importantes y requeridas por los campesinos, será de utilidad para la gestión de los por los campesinos, será de utilidad para la gestión de los bosques en los altos andes.

\section{MATERIALES Y MÉTODOS}

\section{Ámbito de Estudio}

Los bosques nativos estudiados se encuentran por encima de los $3200 \mathrm{~m}$. de altitud en el flanco oriental de la cordillera del Vilcanota, ubicados en la microcuenca de los ríos Patacancha y Málaga, distrito de Ollantaytambo (Provincia de Urubamba) a la que denominamos como zona 1, y en las microcuencas de los ríos Lares y Huarán, distrito de Lares y Calca respectivamente (provincia de Calca) a la que denominamos como zona 2; la ubicación referencial para la zona 1, son las siguientes coordenadas Latitud Sur 13⒈'37', y Latitud Oeste $72^{\circ} 02^{\prime} 04^{\prime \prime}$; la zona 2 , comprendida entre las coordenadas: Latitud Sur 13 $12^{\prime} 01$ ', y Latitud Oeste 7209'27' ( Fig. 1); para el estudio, estos bosques han sido divididas en dos pisos altitudinales; aquellos ubicadas por debajo de los $3500 \mathrm{~m}$. de altitud y otros por encima de ese nivel altitudinal.

Los bosques estudiados se encuentran en el ámbito de las comunidades campesinas que se indican en la Tabla 1 y, el ámbito de estudio y las zonas de trabajo en la Figura 1.

\section{Metodología}

Para las mensuraciones de la oferta de leña de los bosques se emplearon transectos en banda y parcelas con la finalidad de determinar la densidad de las especies y obtener información sobre la oferta de leña; para ello, se identificó el territorio en unidades homogéneas, dependiendo del tipo de vegetación a evaluar: para el matorral espinoso, se utilizó el transecto en banda y, para los bosques mixtos y los de Polylepis, la parcela rectangular o cuadrada. Los transectos lineales utilizados fueron adaptados de Franco et al. (1985), y Bennett y Humprhies (1981), de dos metros de ancho y 25 metros de longitud en razón a la topografía del terreno; en total, se utilizaron 32 transectos. Para delinear la parcela cuadrada o rectangular, dependiendo de la pendiente en el terreno, fueron utilizadas adaptando la propuesta de Barbour et al. (1987). En algunos casos, las parcelas han sido diseñadas a favor de la pendiente otras horizontalmente, dependiendo de la topografía 
y la abundancia de la vegetación a mensurar. Se aplicaron 28 parcelas rectangulares y 12 cuadradas de dimensiones $5 \mathrm{~m} \times 20$ $\mathrm{m} \mathrm{y}, 10 \mathrm{~m} \times 10 \mathrm{~m}$, respectivamente. El objetivo del uso de esta metodología fue para determinar la densidad ecológica; a partir de los resultados, se extrapolaron para la totalidad de los bosques. Para la determinación del área de los bosques y matorrales, se utilizó la información del Proyecto Plantaciones forestales en áreas no intervenidas en las provincias de Calca y Urubamba (GoRe Cusco, 2013). La superficie estimada para cada bosque (ha), la densidad ecológica para la totalidad de las especies evaluadas (árboles/ha); constituyen información para estimar la oferta total de leña, medida en toneladas. Para la obtención del volumen de madera y su densidad se emplearon métodos experimentales en laboratorio; en campo, se midieron la circunferencia basal y distal y, la longitud del fuste. Para la determinación del volumen de leña en los bosques, se mensuraron árboles y arbustos con diámetro superior a $10 \mathrm{~cm}$ a una altura de 1,30 m (MINAGRI-DGFFS, 2012; OSINFORMINAGRI-PCM, 2012), definido como diámetro a la altura del pecho (DAP) utilizado para calcular el volumen del tronco de los árboles, con objeto de obtener la productividad de leña en el bosque (WWF, 2006) y, para la mensuración en los matorrales, la circunferencia basal mínima corresponde a tres $\mathrm{cm}$ o más.

\section{Determinación de Oferta de Leña}

Para la evaluación y posterior estimación de la oferta de biomasa vegetal en los bosques de donde proviene la leña, se consideró que el árbol tiene un fuste cilíndrico truncado, denominado tronco de cono de revolución (Aróstegui, 1970, 1974); determinándose las siguientes variables.
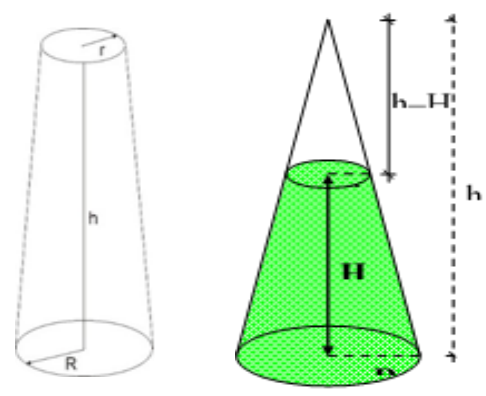

Figura 2. Tronco de cono de revolución utilizado para el cálculo del volumen de biomasa vegetal.

Cálculo del volumen del tronco (leña)

De conformidad a la Figura 2, tenemos:

Donde:

$$
V=\frac{h \pi}{3}\left(R^{2}+r^{2}+R r\right)
$$

$$
\begin{aligned}
& \pi=3,1416 \\
& \mathrm{R}=\text { radio del cono mayor } \\
& \mathrm{r}=\text { radio del cono menor } \\
& \mathrm{h}=\text { altura del tronco de cono }
\end{aligned}
$$

Con las circunferencias medidas en campo, se determinaron los radios ( $r$ y R), siendo la explicación técnica la siguiente:

\section{a. Método analítico.}

Siendo el volumen de la parte sombreada (Fig. 2):

$$
\mathrm{V}=\frac{\mathrm{h} \pi}{3}\left(\mathrm{R}^{2}+\mathrm{r}^{2}+\mathrm{Rr}\right)
$$

Donde:

$$
\begin{aligned}
& \text { radio }=\frac{\mathrm{L}}{2 \pi} \\
& \mathrm{L}=\text { Longitud de la circunferencia basal y distal }
\end{aligned}
$$

\section{b. Método experimental}

Para hallar el volumen del tronco de cono de revolución, se empleó el método de desplazamiento del agua (Chambergo A. 1984).

\section{RESULTADOS}

El estudio de los bosques nativos analizados por encima de $\operatorname{los} 3200 \mathrm{~m}$ de altitud, demuestra que estos ecosistemas ofrecen diversos servicios ecosistémicos de alto valor de uso; la conservación del agua y la producción de leña son vitales para satisfacer demandas en las comunidades. Las comunidades campesinas altoandinas obtienen los recursos para el sustento diario directamente de los bosques nativos, agua y forraje, son obtenidos en un cien por ciento; plantas medicinales en un $95 \%$ y, leña y madera en más del 60\% (Fig. 3).

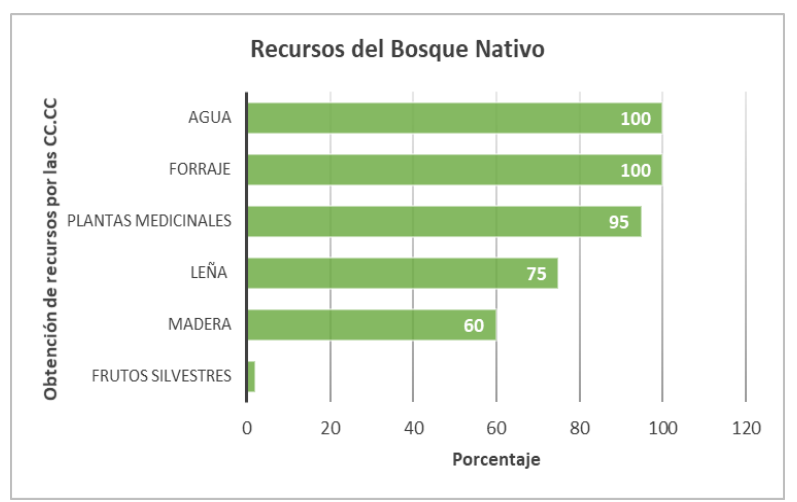

Figura 3. Obtención de Recursos del Bosque por las comunidades campesinas.

Los bosques son fuentes de recursos para las comunidades; sin embargo, actividades como la extracción de leña y madera sin reemplazo, la quema para la agricultura, que deviene en incendios, la introducción de ganado exótico y otros, tienen efectos directos en los bosques que son parches aislados; estas acciones afectan la cobertura vegetal, perturbación que ocasiona impactos ambientales como mayor escorrentía, erosión, disminución de infiltración del agua de precipitación, pérdida de biodiversidad, etc. (Fig. 4)

Cabe resaltar que la provisión de leña, como un servicio ambiental de los bosques nativos es en una proporción del 75 por ciento (Fig. 3).

Además de lo señalado precedentemente, existen impactos directos como la pérdida del suelo por erosión, infiltración escasa del agua de escorrentía produciendo el desecamiento de manantes y una agricultura poco productiva.

\section{Disponibilidad de especies y oferta de leña}

La oferta de leña (t) está en función al volumen, contenido de humedad y densidad de la madera seca. La Tabla 2, evidencia la oferta para cada bosque. 


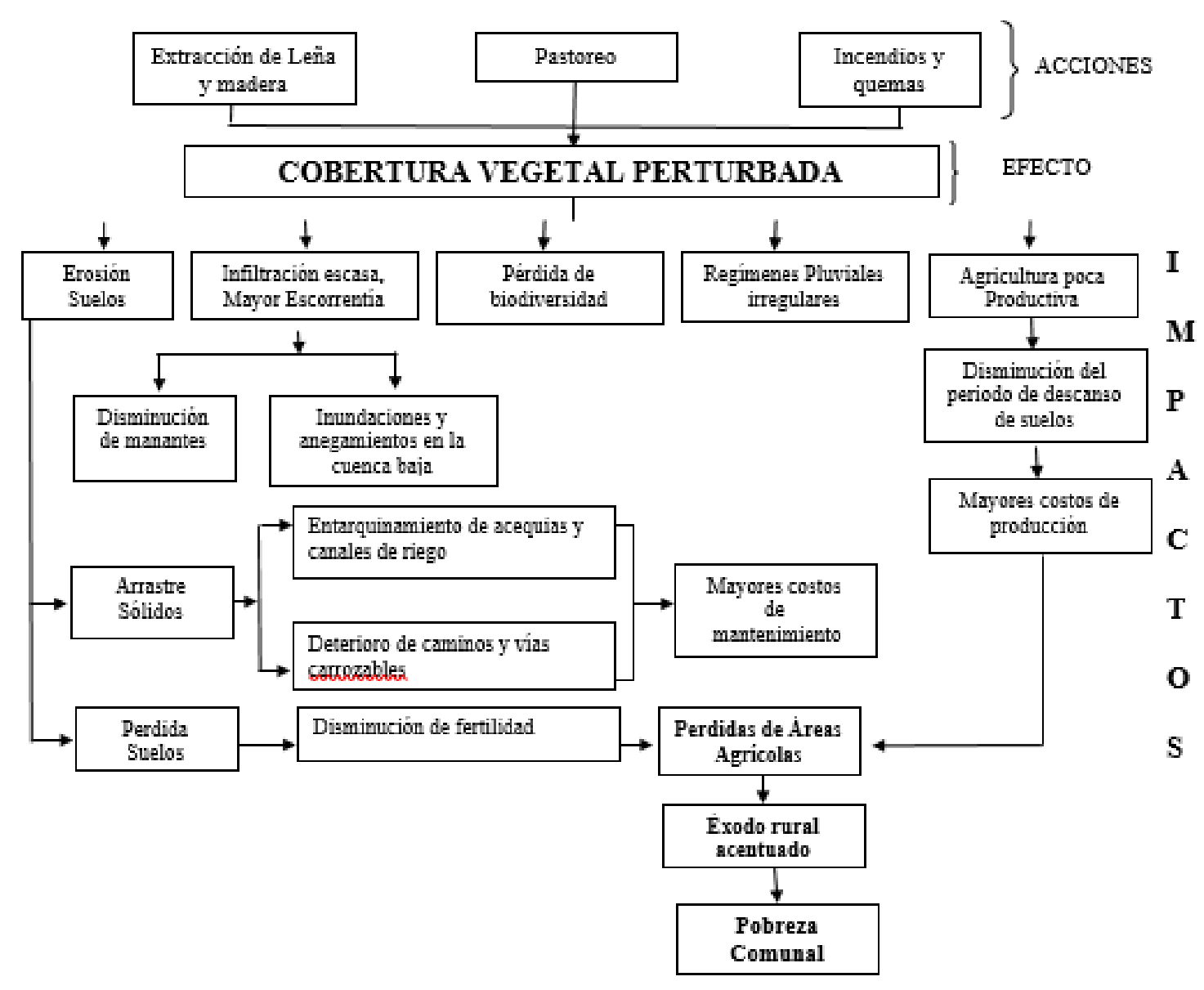

Figura 4. Acciones, efecto e impactos de un mal manejo del bosque.

Los bosques altoandinos evaluados tienen una superficie de 829 ha de los cuales, 510 ha, son bosques mixtos; 159 ha, son bosques de Polylepis; 135 ha corresponden a matorral espinoso y 25 ha, a rodales (Tabla 2). En cuanto a la superficie de los bosques evaluados, el $61,5 \%$ del área total de bosques corresponde a bosques mixtos, el $16,3 \%$ a matorral espinoso, el 19,2\% a bosques de Polylepis y el 3\% a otros.

La oferta de leña calculada en el momento de efectuar la evaluación de campo, alcanza a 178 816,38 toneladas; el matorral espinoso es importante, el camefitismo de las especies posibilita el rebrote y ramificación exuberante, facilitando la obtención de biomasa vegetal como combustible. Los bosques mixtos y matorrales, con 645 ha, constituyen la zona de mayor oferta de leña, poseen una alta productividad y densidad, posibilitando que la comunidad campesina se provea de leña y los excedentes sean comercializados. En efecto, el 58\% de la oferta de leña, proviene del bosque mixto; el $40,2 \%$ de los bosques de Polylepis y, el 1,74\% del matorral espinoso.

En las dos zonas de estudio, la oferta de leña se halla distribuida en la siguiente forma: en la zona uno la superficie evaluada es de 383 ha y presentan una oferta de leña total equivalente a 88199,04 toneladas; mientras que la zona dos tiene una superficie de 446 ha y oferta 90 617,34 toneladas.

Estos bosques, constituyen el mejor ecosistema forestal, por las especies y por una gradación altitudinal secuencial en la distribución espacial de las especies: Alnus acuminata Hunt, Hesperomeles latifolia (Kunth) M. Roem, Escallonia herrerae Mattf, Myrcianthes oreophila (Diels) Mc Vaugh, Hesperomeles heterophylla Hook, Vallea stipularis L.f, Piper elongatum Vahl, Citharexylum argutedentatum Moldenke, Duranta mandonii Moldenke, Escallonia myrtilloides L.f., Gynoxys nítida Muschl. y Polylepis spp.

Los bosques de Polylepis, ubicados por encima de los 3500 m s.n.m. evidencian una mayor densidad, con 950 árboles/ha.

\section{DISCUSIÓN}

Salas (2009), concluye que la extracción de leña y productos forestales no maderables de los bosques nativos andinos de Pacobamba están muy vinculadas con la mujer, mientras que la extracción de madera del bosque nativo es practicada más por los varones que por ellas. Además, este estudio sólo recoge información sobre especies vinculadas a la obtención de leña, más no la oferta; nuestro trabajo evidencia la oferta de leña ( $\mathrm{t} / \mathrm{ha})$; en efecto, el bosque mixto ofrece 366,60 t/ha y, el bosque de Polylepis es altamente productivo, pues su oferta alcanza 424,93 t/ha; el matorral espinoso, puede llegar a ofertar hasta $25 \mathrm{t} / \mathrm{ha}$; esta oferta es de interés para plantear estrategias de conservación mediante reforestación (Tabla 2).

De conformidad a lo indicado por Mejía-Barragán (2011), el 58\% de las familias utilizan leña y gas propano, el $17 \%$ utiliza sólo leña; en cambio nuestro trabajo demuestra que el 100\% de las familias emplean leña proveniente de bosques nativos.

Los bosques altoandinos no sólo ofertan madera y leña como sostienen Ansion \& Van Dam (1986), sino, servicios ecosistémicos como agua, forraje, plantas medicinales, frutos y otros, como señala el presente estudio.

Los estudios de Córdova-Carbajal (2012), señalan que la oferta de leña en las comunidades del Bajo Urubamba es alta y no requieren almacenar, excepto para actividades comunales; 
en cambio, en las comunidades altoandinas, se verificó que las familias almacenan la leña para un periodo mayor a una semana; igualmente, el estudio de Córdova Carbajal, concluye que no es un problema significativo el abastecimiento de leña; en cambio, en las comunidades altoandinas, la oferta de leña proveniente de bosques nativos va disminuyendo, especialmente por encima de los $3500 \mathrm{~m}$ s.n.m.

Delgado (2003), evalúa los bosques de Manto (Lares) especificando la flora arbórea utilizada, más no determina la oferta de leña de dichos bosques; nuestro trabajo especifica las especies utilizadas y la oferta total de leña.

Alonso et al., (2001), sostienen que en la región de los Andes orientales sólo queda el $25 \%$ de la cubierta forestal original y sólo el $25 \%$ de sus bosques actuales cuenta con algún tipo de protección. Esta situación hace que la Cordillera de los Andes sea una de las regiones más importantes para la conservación de la biodiversidad en el mundo; nuestros estudios confirman esta aseveración y nos parece que incluso existe un porcentaje menor de la cubierta vegetal original, pues los bosques nativos constituyen parches en el ámbito de estudio. Los bosques nativos en los altos andes, no sólo están siendo afectados por la sobre extracción de leña como sostiene MINAGRI (2012); nuestros estudios ratifican esta conclusión $\mathrm{y}$, además indican otras connotaciones ambientales que afectan los servicios ecosistémicos.

Análogamente a lo reportado por Ocaña (1994), en las comunidades estudiadas que se hallan ubicadas por encima de los 3600 o $4000 \mathrm{~m}$ de altitud el déficit de material energético es crítico y no llega a abastecer las necesidades más urgentes, especialmente si de leña se trata; por lo tanto, es substituido por otros elementos como la bosta de ganado (vacuno, ovino y camélidos sudamericanos) generados en la propia comunidad y/o la leña de eucalipto, proveniente de otras zonas.

Pretell et al. (1985), Reynel et al. (2006), concluyen que las especies forestales son de alta utilidad en el control de erosión y en la oferta de biomasa vegetal; nuestros estudios conducen a esta conclusión y señalan que las especies maderables, además de ofertar leña brindan otros servicios ambientales.

Desde hace décadas se observa la creciente escasez de leña para uso doméstico, tal como lo sostienen Córdova-Aguilar (1992), Ektvedt (2011) y MINAGRI (2012); en efecto, esta conclusión es ratificada por nuestros trabajos de campo y especifican una oferta escasa de leña de los bosques altoandinos, especialmente para comunidades ubicadas por encima de los $3500 \mathrm{~m}$.

Los estudios de campo demuestran que los bosques altoandinos están en franco proceso de retraimiento debido a las actividades que generan impactos sobre la biomasa vegetal y que las comunidades campesinas requieren apoyo del estado para proteger sus bosques.

\section{CONCLUSIONES}

Los bosques altoandinos son altamente productivos, brindan servicios ecosistémicos múltiples, aspecto que posibilita su conservación. En el ámbito de estudio, el bosque mixto ocupa el $61,5 \%$ de la superficie de bosques y su oferta es del $58 \%$ del total de leña utilizada por las comunidades. Los bosques monoespecíficos de Polylepis constituyen el 19,2\% del total del bosque en el ámbito estudiado y, la oferta de leña es de $40,2 \%$. El matorral espinoso constituye una fuente importante de leña para las comunidades; su extensión es inferior al 16,3\% del total de bosques y proporciona el 1,74\% de leña; empero, todas las especies tienen preferencia alta.

El material energético de la biomasa vegetal, corresponde a vegetación arbustiva y arbórea de los bosques; existe mayor diversidad en los bosques y bosquetes inferiores a $3500 \mathrm{~m}$. de altitud, constituyendo bosques mixtos, matorrales espinosos y asocies interespecíficos; en cambio, a altitudes superiores a los $3500 \mathrm{~m}$ se observan rodales dominados por los géneros Baccharis, Polylepis, Gynoxis, Weinmannia, Cytharexilum, Buddleja.

De las especies más utilizadas en el abastecimiento de leña, destacan por su abundancia los géneros Hesperomeles, Buddleja, Escallonia y Polylepis; sin embargo, considerando la biomasa que ofertan, las especies más importantes pertenecen a los géneros Alnus, Acalipha, Baccharis, Myrica, Berberis, Duranta, Barnadesia, Gynoxis, Hesperomeles, Myrcianthes, Citharexylum, Escallonia y Polylepis.

En las comunidades ubicadas entre 3200 y $3500 \mathrm{~m}$ de altitud; los bosques mixtos, matorrales espinosos y asocies interespecíficos, son los que proporcionar madera y leña en cantidades aún suficientes; en cambio en las comunidades localizadas por encima de los $3500 \mathrm{~m}$ de altitud las especies de Polylepis son las que proveen las mayores ofertas de leña. Las comunidades campesinas altoandinas poseen predisposición para la reforestación y están dispuestos a participar en programas forestales.

Las especies del género Polylepis, son de interés comunal, es apreciado como combustible, para obtener carbón, madera y como tintórea.

El bosque ofrece un mayor número de servicios ecosistémicos. Análogamente, en las comunidades más altas, la vegetación es escasa, con menor presencia de matorral espinoso, por lo que emplean bosta de ganado vacuno, ovino y de camélidos sudamericanos; la oferta de leña es menor. En comunidades ubicadas a altitudes superiores a los $3500 \mathrm{~m}$, el recurso más importante de leña es el bosque de Polylepis.

Los habitantes de las zonas altoandinas, conservan una relación directa con su entorno, están dedicados a la agricultura y ganadería y utilizan la leña como un servicio ambiental. Esta situación está en un momento de cambio, por la necesidad de algunos de buscar fuentes de trabajo en la ciudad y por el turismo vivencial que, de no administrarse adecuadamente, puede permear la cultura tradicional del uso de leña.

Los impactos más resaltantes, consecuencia de la extracción de leña son: la erosión de suelos, infiltración escasa, mayor escorrentía, regímenes pluviales irregulares, agricultura poca productiva, pérdida de biodiversidad, disminución de manantes, arrastre de sólidos, pérdida de la fertilidad de los suelos, con consecuencias en la economía campesina generando un éxodo rural y pobreza comunal.

La oferta total de leña calculada para los bosques altoandinos de la cordillera del Vilcanota es de 178 816,38 toneladas; esta oferta calculada para las dos zonas de estudio; la zona uno oferta 88 199,04 toneladas; mientras que la zona dos tiene una superficie de 446 ha y oferta 90 617,34 toneladas.

\section{AGRADECIMIENTOS}

A Ecosistemas Andinos-ECOAN, por posibilitar la ejecución del estudio y, al colega Alfredo Tupayachi Herrera por el apoyo en la identificación de las especies botánicas.

\section{REFERENCIAS BIBLIOGRÁFICAS}

Alonso, L.E., Alonso, A., Schulenberg, T.S. \& Dallmeier, F. (2001). Biological and social assessments of the Cordillera del Vilcabamba, Peru. Washington D.C., United States of America. 296 p. RAP Working Papers $\mathrm{N}^{\circ} 12$ and SI/MAB series $\mathrm{N}^{\circ}$ 6. Conservation International.

Ansión, J. \& Van Dam, C. (1986). El árbol y el bosque en la sociedad andina. Lima, Perú, Talleres Gráficos Art. Lantrec. $119 \mathrm{p}$. 
Aróstegui, A. (1970). Estudio de las propiedades físicomecánicas de 16 especies maderables del país. Lima, Perú, Centro de investigaciones forestales. v.2, $76 \mathrm{p}$.

Aróstegui, A. (1974). Estudio tecnológico de maderas del Perú: Características tecnológicas y usos de la madera de 145 especies del país. Lima, Perú, Ministerio de Agricultura. 483 p.

Aróstegui, A. (1982). Recopilación y análisis de estudios tecnológicos de maderas peruanas. Documento de trabajo $\mathrm{N}^{\circ}$ 2. Proyecto PNUD/FAO/PER/81/002. Lima, Perú. 57 p.

Barbour, M.G., J. H. Burk \& Pitts, W.D. (1987). Terrestrial Plant Ecology. Second Edition. The-Benjamin/Cummings Publishing Company, California, 634 pp.

Bennett, D. \& Humphries, D. (1981). Introducción a la ecología de campo. Edit. H. Blume. Madrid. España.

Bermejo, J. \& Passeti, F. (1985). El árbol en apoyo de la Agricultura. Documento de trabajo $\mathrm{n}^{\circ}$ 4. Proyecto FAO/Holanda/INFOR. Lima, Perú. 44 p.

Chambergo, A. (1984). Influencia de los elementos xilemáticos en las propiedades físico-mecánicas de 45 maderas del Perú. Tesis Ing. Forestal, UNALM. Lima, Perú, UNALM. 110 p.

Córdova-Aguilar, H. (1992). Firewood use and the effect on the ecosystem: A Case study of the sierra of Piura, northwestern Peru. GeoJournal, 26(3): 297-309.

Córdova-Carbajal, P. (2012). Estudio del consumo de leña en dos comunidades nativas de la cuenca del rio Bajo Urubamba. UNA La Molina. Lima. 73 p.

Delgado, M. del C. \& Dueñas, N.R. (2003). Composición y diversidad florística del bosque nuboso: Localidad de Manto - Lares. Calca. Tesis Biólogo. UNSAAC. 129 p.

Dirección General de Flora y Fauna Silvestre-Ministerio de Agricultura- MINAG-DGFFS (2012). Anuario Forestal. Disponible en http://dgffs.minag.gob.pe/pdf/estadistica_forestal/anuarios/A NUARIO_PERU_FORESTAL_2009.pdf.

Ehrhardt-Martinez, K. (1998). Social determination of deforestation in developing countries: A cross-national study. Social Forces Vol. 77, No. 2 (Dec., 1998), pp. 567-586. Published by: Oxford University Press.

Ektvedt TM. (2011). Firewood consumption amongst poor inhabitants in a semiarid tropical forest: A case study from Piura, northern Peru. Norsk Geografisk Tidsskrift Norwegian Journal of Geography 65(1): 28-41.

FAO. (1981). Mapa de la situación en materia de leña en los países en desarrollo. Roma.

FAO. (1983). Disponibilidad de leña en los países en desarrollo. Estudio FAO Montes 42. Roma Italia. Disponible en: www.fao.org/3/x5329s/x5329s02.htm

Geist, H.J. \& Lambin F. (2001). What drives tropical deforestation? A meta-analysis of proximate and underlying causes of deforestation based on subnational case study evidence. Louvain-la-Neuve, Belgium, University of Louvain. 110 p. LUCC Report Series n. ${ }^{\circ} 4$.

Franco, F., De La Cruz, G., Rocha, A., Navarrete, N., Flores, G., Kato, E., Sánchez, S., Abarca, L. \& Bedia, C. (1985). Manual de ecología. Edit. Trillas S.A. Segunda edición. México D.F., México.

Gil-Mora, J.E. (2002). Deforestación: Principales causas y efectos ambientales. Revista Oropesa (43): 20-23.
Gil-Mora, J.E., Baca-Flores, J.A. \& Álvarez-Moscoso, M.E. (2019). Consumo de leña y estimaciones del valor calorífico de especies de plantas utilizadas por las comunidades campesinas de la cordillera del Vilcanota, Cusco. Revista Queuña, 11(1): 41-58.

Gobierno Regional Cusco. Gerencia Regional de Planeamiento, Presupuesto y Acondicionamiento Territorial. Área Funcional de Estudios de pre Inversión. (2013). Proyecto de instalación de plantaciones forestales en áreas no intervenidas en las provincias de Calca y Urubamba de la Región Cusco. AFEP. 96 p.

Loján, L. (1992). El verdor de los Andes. Proyecto Desarrollo Forestal Participativo en los Andes. Quito, Ecuador, Editora Luz de América. 217 p.

Mejía-Barragán, F. (2011). Implicaciones ambientales del uso de leña como combustible doméstico en la zona rural de Usme. Tesis Mg. en Medio Ambiente y Desarrollo. Bogotá, Universidad Nacional de Colombia 119 p.

MINAGRI (Ministerio de Agricultura y Riego, Perú); Dirección General de Flora y Fauna Silvestre. (2012). Anuario Forestal (en línea). Lima, Perú. 164 p. Consultado 5 jun. 2012. Disponible en http://dgffs.minag.gob.pe/pdf/estadistica_forestal/anuarios/A NUARIO_PERU_FORESTAL_2011.pdf.

MINAGRI-OSINFOR-PCM. (2012). Protocolo para la evaluación de individuos maderables. Lima.13 p.

Ministerio de Agricultura-Dirección General Forestal y de Fauna Silvestre. (2012). Manual base para la Planificación y Ejecución de Inventarios Forestales. Lima. 52 p.

Ministerio de Energía y Minas-MEM. (2010). Balance Nacional de Energía. Lima. Disponible en: http://www.minem.gob.pe/publicacion.php?idSector=12\&id Publicacion=418 (acceso 5 junio 2012).

Ocaña, D. (1994). Desarrollo forestal campesino en la región Andina del Perú. Lima, Perú, Ministerio de Agricultura. 218 p.

Pretell, J., Ocaña, D. \& Barahona, E. (1985). Apuntes sobre algunas especies forestales de la sierra peruana. Proyecto FAO-Holanda/INFOR. Lima, Perú. 120 p.

Reynel, C., Pennington, T.D., Pennington, R.T., Marcelo, J.L. \& Daza, A. (2006). Árboles útiles del Ande peruano: una guía de identificación, ecología y propagación de las especies de la sierra y los bosques montanos del Perú. Talleres Gráficos de Tarea Asociación Gráfica Educativa. 462 p. Lima, Perú.

Salas-Laines, R. (2009). Diagnóstico con enfoque de género sobre el uso de los bosques nativos andinos de PacobambaApurímac. Tesis para optar el Título de Ingeniero Forestal. Universidad Agraria La Molina. Lima. 127 p.

World Wildlife Fund- WWF. (2006). Manual de Censos Forestales, Lima, Perú. 72 p.
Presentado: 05/04/2021

Aceptado: 27/05/2021

Publicado: 19/07/2021 


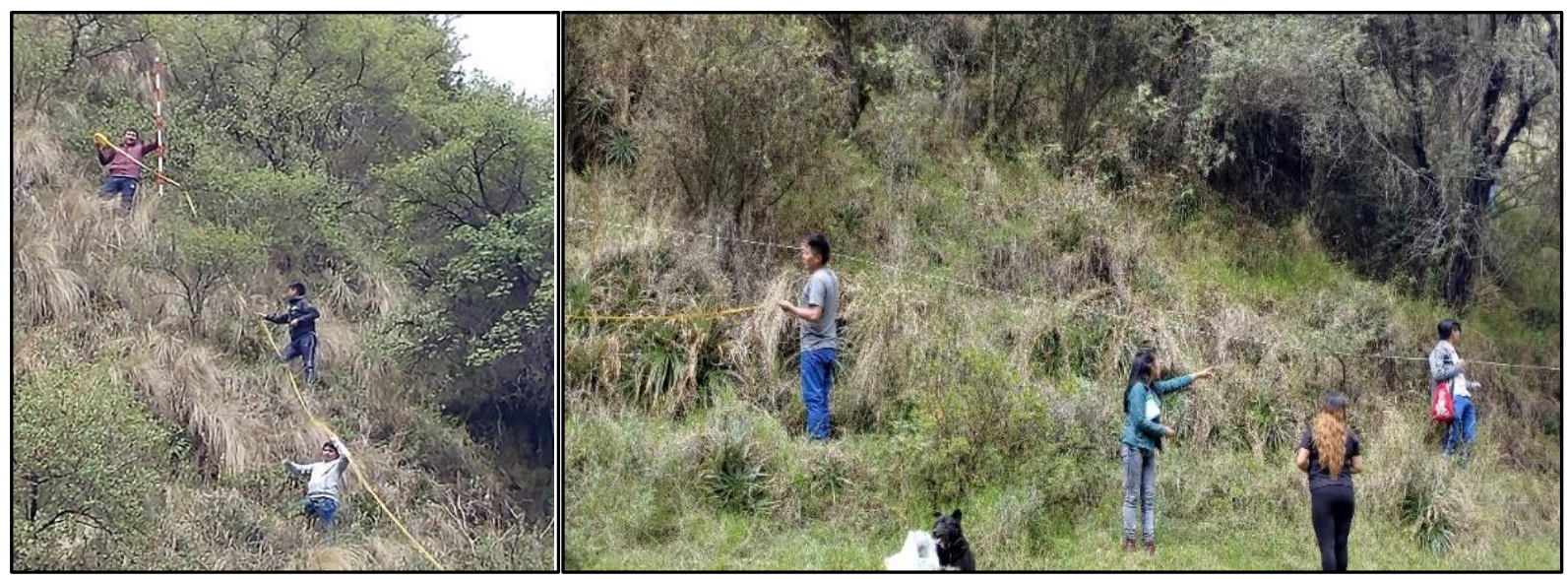

Figura 5. Demarcando parcelas para la evaluación de la oferta de leña.

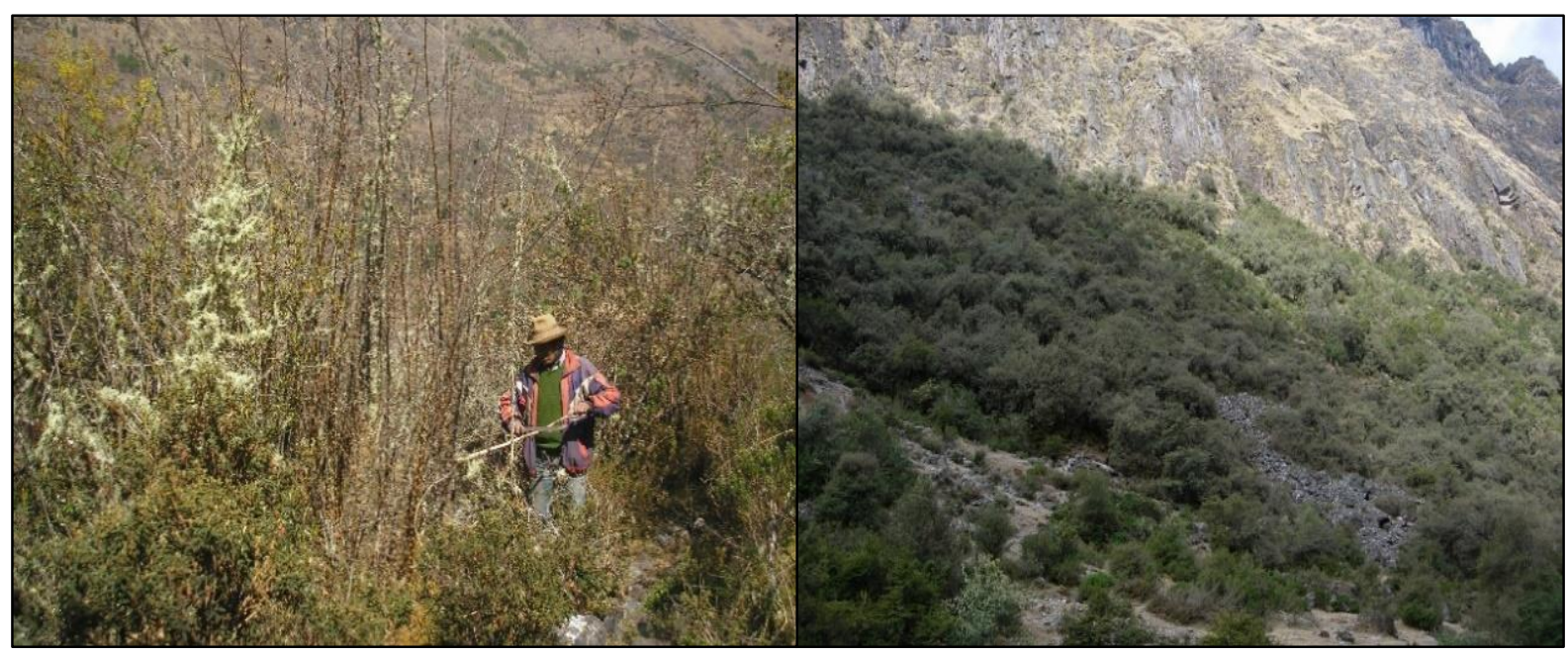

Figura 6. El matorral espinoso y los bosques monoespecíficos de Polylepis constituyen ecosistemas con servicios ambientales importantes para las comunidades campesinas altoandinas.

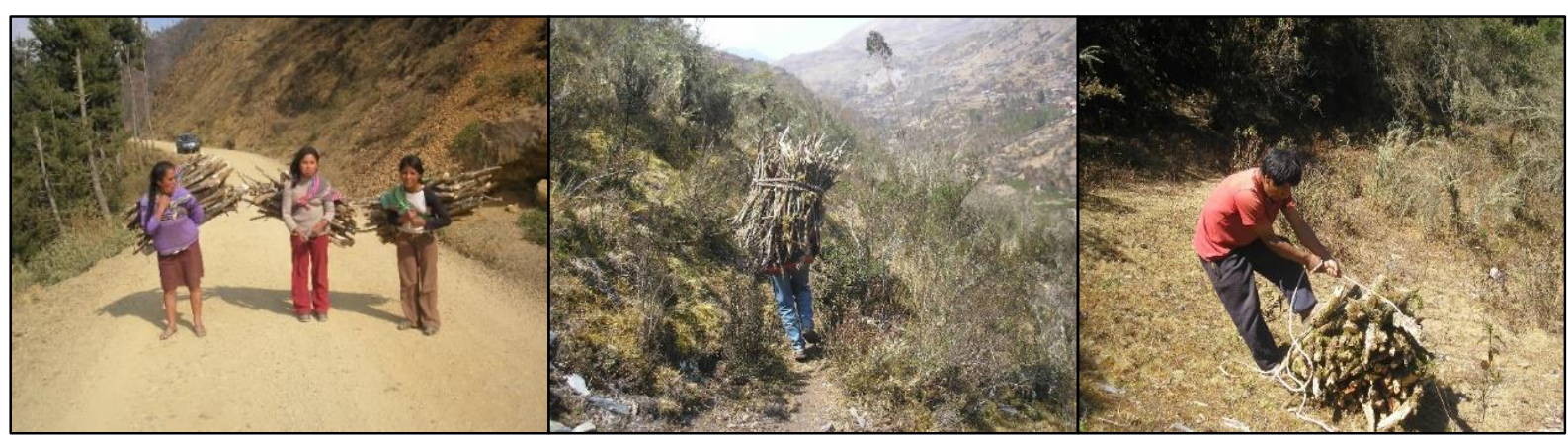

Figura 7. Mujeres y varones obtienen leña. Las mujeres para la cocción diaria y los varones para las actividades de la semana. 


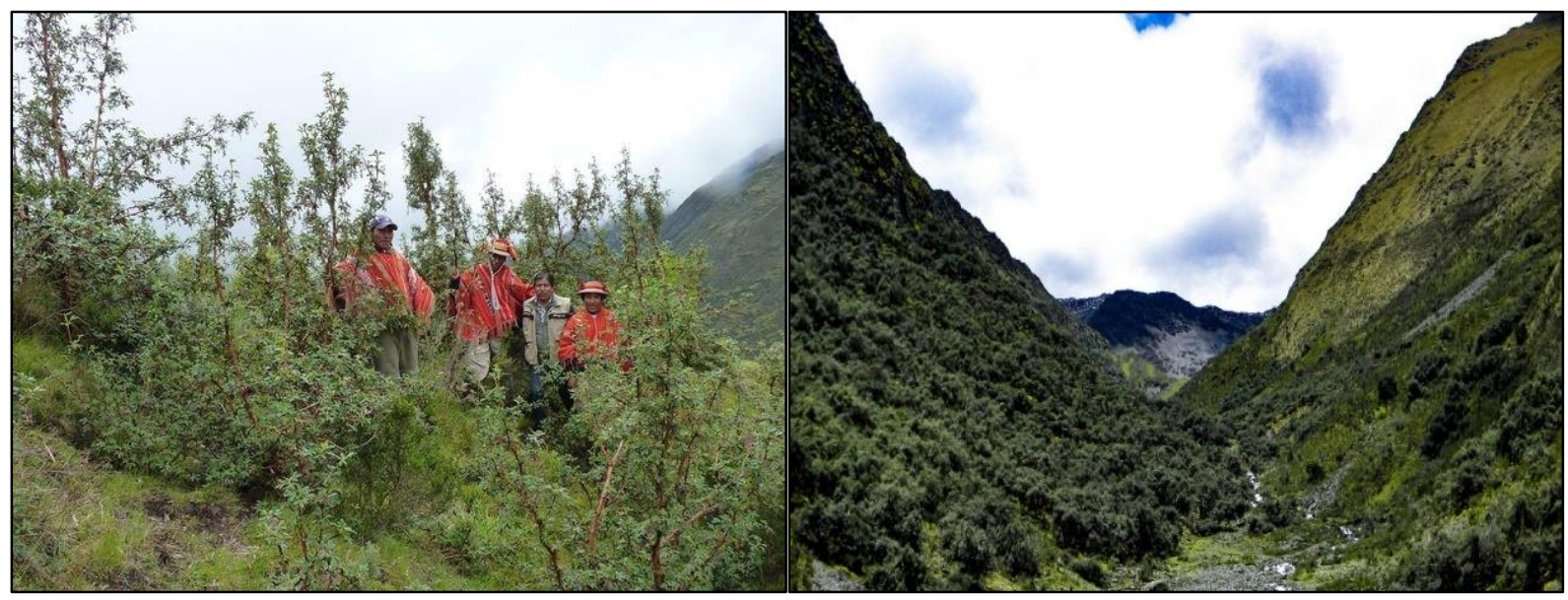

Figura 8. Por debajo de los $3500 \mathrm{~m}$ s.n.m. los bosques montanos forman una cubierta vegetal importante y por encima de ese nivel altitudinal, predominan los bosques de Polylepis

Tabla 1: Ubicación de bosques y comunidades estudiadas

\begin{tabular}{|c|c|c|c|c|}
\hline $\begin{array}{l}\text { Microcuenca y } \\
\text { Zona de estudio }\end{array}$ & Río & Comunidad campesina & $\begin{array}{l}\text { Altitud, } \\
\text { m s.n.m. }\end{array}$ & Orientación referencial \\
\hline Málaga Zona 1 & Málaga & T'astayoq-Abra de Málaga & 4350 & NNE respecto de Ollantaytambo \\
\hline Zona 1 & Patacancha & $\begin{array}{l}\text { Phallata } \\
\text { Willoq } \\
\text { Rumira-Sondormayu } \\
\text { Patacancha } \\
\end{array}$ & $\begin{array}{l}3250 \\
3950 \\
4000 \\
4050 \\
\end{array}$ & $\begin{array}{l}\text { ENE respecto de Ollantaytambo } \\
\text { ENE respecto de Ollantaytambo } \\
\text { ENE respecto de Ollantaytambo } \\
\text { ENE respecto de Ollantaytambo }\end{array}$ \\
\hline Warán Zona 2 & $\begin{array}{ll}\text { Warán } & \mathrm{y} \\
\text { Canchacancha }\end{array}$ & $\begin{array}{l}\text { Canchacancha } \\
\text { T'aqllapata }\end{array}$ & $\begin{array}{l}3700 \\
3550\end{array}$ & $\begin{array}{l}\text { NNE respecto de la ex hacienda Warán. } \\
\text { ENE respecto de la ex hacienda Warán; margen } \\
\text { Izquierda del río Warán. }\end{array}$ \\
\hline \multirow[t]{2}{*}{ Lares Zona 2} & Lares & $\begin{array}{l}\text { Pampacorral } \\
\text { Kiswarani }\end{array}$ & $\begin{array}{l}3400 \\
3600 \\
\end{array}$ & $\begin{array}{l}\text { SSE de Lares sobre la margen izquierda del río } \\
\text { Maukao. } \\
\text { S de Lares a la margen izquierda del río Maukao. }\end{array}$ \\
\hline & Trapiche & $\begin{array}{l}\text { Tambowaylla } \\
\text { Wakawasi } \\
\text { Kunkani }\end{array}$ & $\begin{array}{l}3400 \\
3800 \\
3800\end{array}$ & $\begin{array}{l}\text { W respecto de Lares, sobre la margen derecha del } \\
\text { río Trapiche } \\
\text { W respecto de Lares, entre los ríos Pukamayu y } \\
\text { Kurukumayu. } \\
\text { WSW de Lares sobre la margen derecha del río } \\
\text { Kunkani }\end{array}$ \\
\hline
\end{tabular}

Fuente: Elaborado en base a la Carta Nacional y trabajos de campo.

Tabla 2. Oferta de leña en los principales bosques comunales estudiados. Basado en observaciones y mensuraciones de campo.

\begin{tabular}{|c|c|c|c|c|c|c|c|c|}
\hline $\begin{array}{l}\text { Bosque } \\
\text { y zona } \\
\text { de } \\
\text { estudio }\end{array}$ & C.C & $\begin{array}{l}\text { Altitud, } \\
\text { m.s.n.m }\end{array}$ & $\begin{array}{lll}\text { Tipología y } & \text { especies } \\
\text { representativas } & \end{array}$ & $\begin{array}{l}\text { Super } \\
\text { ficie (ha) }\end{array}$ & $\begin{array}{l}\text { Densidad } \\
\text { Ecológica } \\
\text { (árboles/ha) }\end{array}$ & $\begin{array}{l}\text { Oferta } \\
\text { Biomasa } \\
\left(\mathrm{m}^{3} / \mathrm{ha}\right) *\end{array}$ & $\begin{array}{l}\text { Oferta } \\
\text { leña } \\
(\mathrm{t} / \mathrm{ha})\end{array}$ & $\begin{array}{l}\text { Oferta total } \\
\text { de leña }(t)\end{array}$ \\
\hline $\begin{array}{l}\text { Choque } \\
\text { Chaka } \\
\text { Zona } 1\end{array}$ & Phallata & $\begin{array}{l}3500- \\
4600\end{array}$ & $\begin{array}{l}\text { Bosque mixto con predominio } \\
\text { de Alnus acuminata, } \\
\text { Myrcianthes oreophila, } \\
\text { Hesperomeles latifolia, } \\
\text { Citharexylum argutedentatum, } \\
\text { Escallonia herrerae; en la parte } \\
\text { alta Polylepis. }\end{array}$ & $\begin{array}{l}\text { A ambos } \\
\text { flancos } \\
\text { del río } \\
\text { Yuraqma } \\
\text { yu. } 190 \\
\text { ha. }\end{array}$ & 650 & 832,65 & 366,60 & 69654,0 \\
\hline $\begin{array}{l}\text { Camino } \\
\text { a } \\
\text { Choque } \\
\text { Chaka } \\
\text { Zona 1 }\end{array}$ & Phallata & $\begin{array}{l}3300- \\
3500\end{array}$ & $\begin{array}{l}\text { Matorral espinoso. Barnadesia } \\
\text { berberoides, Berberis carinata, } \\
\text { Berberis commutata, } \\
\text { Kageneckia lanceolata, } \\
\text { Dasyphyllum leiocephalum. } \\
\text { Lycianthes lycioides. }\end{array}$ & 15 & 1300 & 10,79 & 3,12 & 46,80 \\
\hline $\begin{array}{l}\text { Laderas } \\
\text { de } \\
\text { Willoq } \\
\text { Zona } 1\end{array}$ & Willoq & 3950 & $\begin{array}{l}\text { Matorral espinoso. Baccharis } \\
\text { buxifolia, Baccharis odorata, } \\
\text { Berberis carinata, Barnadesia } \\
\text { berberoides, Duranta mandonii, } \\
\text { Dunalia spinosa. }\end{array}$ & 08 & 200 & 0,36 & 0,24 & 1,92 \\
\hline $\begin{array}{l}\text { Queuña } \\
\text { qocha }\end{array}$ & $\mathrm{Wi}$ & $\begin{array}{l}4150- \\
4600\end{array}$ & $\begin{array}{l}\text { Bosque Monoespecífico con } \\
\text { predominio de Polylepis, }\end{array}$ & 60 & 600 & 234,00 & 105,48 & 6328,80 \\
\hline
\end{tabular}




\begin{tabular}{|c|c|c|c|c|c|c|c|c|}
\hline Zona 1 & $\begin{array}{l}\text { lloq, } \\
\text { Pata } \\
\text { cancha }\end{array}$ & & $\begin{array}{l}\text { eventualmente } \quad \text { Escallonia } \\
\text { myrtilloides. }\end{array}$ & & & & & \\
\hline $\begin{array}{l}\text { Alturas } \\
\text { de } \\
\text { Rumira } \\
\text { - Sondor } \\
\text { Zona 1 }\end{array}$ & $\begin{array}{l}\text { Rumira } \\
- \\
\text { Sondor }\end{array}$ & $\begin{array}{l}4000- \\
4400\end{array}$ & $\begin{array}{l}\text { Rodales de Baccharis odorata, } \\
\text { Baccharis buxifolia y Baccharis } \\
\text { latifolia, a lo largo del río y el } \\
\text { tramo carretero. }\end{array}$ & 25 & 120 & 21,456 & 0,288 & 7,20 \\
\hline $\begin{array}{l}\text { Saywa y } \\
\text { Queuña } \\
\text { Zona } 1\end{array}$ & $\begin{array}{l}\text { Rumira } \\
- \\
\text { Sondor }\end{array}$ & $\begin{array}{l}3800- \\
4100\end{array}$ & 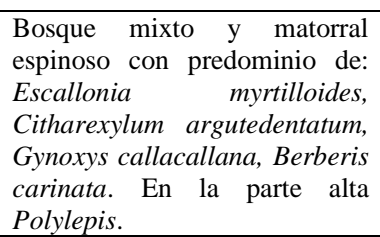 & 20 & $\begin{array}{l}\text { En el caso } \\
\text { de Saywa } \\
250 \text { y en el } \\
\text { caso del } \\
\text { bosque de } \\
\text { Polylepis } \\
180\end{array}$ & $\begin{array}{l}249,225 \\
70,20\end{array}$ & $\begin{array}{l}123,70 \\
31,644\end{array}$ & $\begin{array}{l}2474,00 \\
634,88\end{array}$ \\
\hline $\begin{array}{l}\text { Málaga } \\
\text { Zona } 1\end{array}$ & $\begin{array}{l}\text { T'asta } \\
\text { yoq }\end{array}$ & $\begin{array}{l}3700- \\
4300\end{array}$ & $\begin{array}{l}\text { Bosque mixto con predominio } \\
\text { de Polylepis, Escallonia } \\
\text { herrerae, } \quad \text { Escallonia } \\
\text { myrtilloides, Gynoxys nítida, } \\
\text { Citharexylum argutedentatum; } \\
\text { en la parte alta Polylepis. }\end{array}$ & 65 & $\begin{array}{l}280 \text { en el } \\
\text { bosque } \\
\text { mixto; en el } \\
\text { bosque de } \\
\text { Queuña, la } \\
\text { densidad } \\
\text { alcanza a } \\
152\end{array}$ & 279,132 & 0,7128 & 46,337 \\
\hline $\begin{array}{l}\text { Bosque } \\
\text { montano } \\
\text { Zona } 2\end{array}$ & $\begin{array}{l}\text { Parte } \\
\text { baja del } \\
\text { río Wa } \\
\text { ran }\end{array}$ & $\begin{array}{l}2800- \\
3400\end{array}$ & $\begin{array}{l}\text { Bosque mixto: Caesalpinia } \\
\text { spinosa, Schinus molle, } \\
\text { Weinmannia pentaphylla, Alnus } \\
\text { acuminata, Escallonia resinosa, } \\
\text { Acalypharanioides, } \\
\text { Hesperomeles heterophylla. }\end{array}$ & 95 & 100 & 36,08 & 14,85 & 1410,75 \\
\hline $\begin{array}{l}\text { Bosque } \\
\text { seco } \\
\text { montano } \\
\text { Zona } 2\end{array}$ & $\begin{array}{l}\text { Camino } \\
\text { a Can } \\
\text { Cancha } \\
\text { cancha } \\
\text { a ambos } \\
\text { flancos } \\
\text { del río } \\
\text { Waran }\end{array}$ & $\begin{array}{l}3400- \\
3750\end{array}$ & 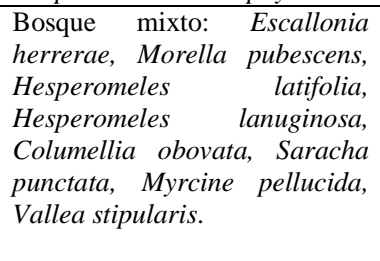 & 120 & 330 & 328,977 & 163,28 & 19593,6 \\
\hline $\begin{array}{l}\text { Matorral } \\
\text { mixto } \\
\text { Zona } 2\end{array}$ & $\begin{array}{l}\text { Camino } \\
\text { a } \\
\text { Cancha } \\
\text { cancha, } \\
\text { hacia } \\
\text { las } \\
\text { laderas } \\
\text { del piso } \\
\text { de valle }\end{array}$ & $\begin{array}{l}3400- \\
3750\end{array}$ & $\begin{array}{l}\text { Matorral espinoso: Puya } \\
\text { herrerae, Berberis carinata, } \\
\text { Berberis boliviana, Barnadesia } \\
\text { berberoides, Dunalia spinosa, } \\
\text { Escallonia herrerae, Vallea } \\
\text { stipularis, Duranta mandonii. }\end{array}$ & $22, .5$ & 250 & 249,225 & 123,70 & 2783.25 \\
\hline $\begin{array}{l}\text { T'asta } \\
\text { monte } \\
\text { Zona } 2\end{array}$ & $\begin{array}{l}\text { Camino } \\
\text { a } \\
\text { Cancha } \\
\text { cancha }\end{array}$ & $\begin{array}{l}3700- \\
3900\end{array}$ & $\begin{array}{lr}\text { Bosque mixto: } & \text { Escallonia } \\
\text { mirtylloides, } & \text { Gynoxys } \\
\text { longifolia, Vallea } & \text { stipularis, } \\
\text { Citharexylum argutedentatum. }\end{array}$ & 20 & 97 & 96,6993 & 47,99 & 959,80 \\
\hline $\begin{array}{l}\text { Cancha } \\
\text { cancha } \\
\text { Zona } 2\end{array}$ & $\begin{array}{l}\text { Cancha } \\
\text { cancha }\end{array}$ & $\begin{array}{l}3850- \\
4100\end{array}$ & $\begin{array}{l}\text { Bosque monoespecífico de } \\
\text { Polylepis; } \quad \text { eventualmente } \\
\text { Gynoxys nítida. }\end{array}$ & 25 & 944 & 4448,88 & 2005,62 & 50140,5 \\
\hline $\begin{array}{l}\text { Sutoq } \\
\text { Zona } 2\end{array}$ & $\begin{array}{l}\text { Kunka- } \\
\text { ni }\end{array}$ & $\begin{array}{l}3950- \\
4300\end{array}$ & $\begin{array}{l}\text { Monoespecífico de Polylepis, } \\
\text { con escasa presencia de } \\
\text { Gynoxys nítida. }\end{array}$ & 18 & 400 & 1,56 & 1,00 & 18,00 \\
\hline $\begin{array}{l}\text { Wachu } \\
\text { monte } \\
\text { Zona } 2\end{array}$ & $\begin{array}{l}\text { Pampa } \\
\text { corral }\end{array}$ & 3500 & $\begin{array}{l}\text { Matorral espinoso: } \text { Berberis } \\
\text { commutata, Berberis, carinata, } \\
\text { Barnadesiar berberoides, } \\
\text { Durantarandonii, } \\
\text { Citharexylum argutedentatum, } \\
\text { Saracha punctata; en la parte } \\
\text { alta, Escallonia myrtilloides. }\end{array}$ & 30 & 135 & 1,12 & 0,324 & 9,72 \\
\hline $\begin{array}{l}\text { Wesqan } \\
\text { a } \\
\text { Queuña } \\
\text { Zona } 2\end{array}$ & $\begin{array}{l}\text { Pampa } \\
\text { corral }\end{array}$ & 4100 & Monoespecifico de Polylepis & 12 & 760 & 296,0 & 133,60 & 1603,20 \\
\hline $\begin{array}{l}\text { Kiswa } \\
\text { rani } \\
\text { Zona } 2\end{array}$ & $\begin{array}{l}\text { Kiswa } \\
\text { rani }\end{array}$ & 3700 & 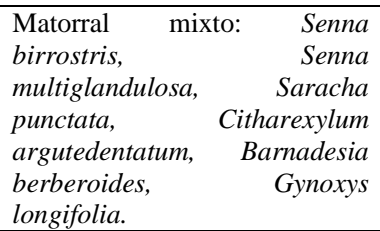 & 12,5 & 200 & 35,76 & 19,84 & 248,00 \\
\hline
\end{tabular}




\begin{tabular}{|c|c|c|c|c|c|c|c|c|}
\hline $\begin{array}{l}\text { Hatum } \\
\text { Queuña } \\
\text { Zona 2 }\end{array}$ & $\begin{array}{l}\text { Kiswa } \\
\text { rani }\end{array}$ & $\begin{array}{l}3900- \\
4100\end{array}$ & Monosespecífico de Polylepis & 14 & 450 & 175,5 & 79,11 & 1107,54 \\
\hline $\begin{array}{l}\text { Tambo } \\
\text { Waylla } \\
\text { Zona } 2\end{array}$ & $\begin{array}{l}\text { Tambo } \\
\text { waylla }\end{array}$ & 3400 & 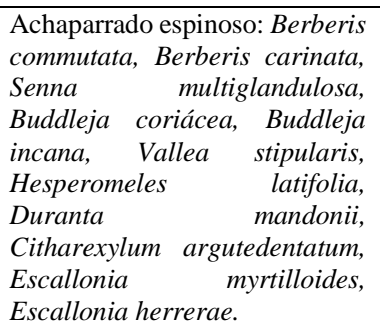 & 25 & 180 & 1,15 & 0,39 & 9,75 \\
\hline $\begin{array}{l}\text { Chanka } \\
\text { Pampa } \\
\text { Zona } 2\end{array}$ & $\begin{array}{l}\text { Waka } \\
\text { wasi }\end{array}$ & 3750 & $\begin{array}{l}\text { Matorral espinoso: } \text { Barnadesia } \\
\text { berberoides, } \\
\begin{array}{l}\text { leiocephasyphylum, } \\
\text { odorata, Durcharis } \\
\text { Citharexylum argutedentatum. }\end{array}\end{array}$ & 22 & 120 & 0,99 & 0,288 & 6,33 \\
\hline $\begin{array}{l}\text { Mantana } \\
\text { y } \\
\text { Zona } 2\end{array}$ & $\begin{array}{l}\text { Waka } \\
\text { wasi }\end{array}$ & $\begin{array}{l}3900- \\
4400\end{array}$ & $\begin{array}{l}\text { Bosque de Polylepis, con } \\
\text { presencia de Gynoxys nitida, } \\
\text { Escallonia Myrtilloides. }\end{array}$ & 30 & 950 & 929,95 & 424,93 & 12726,90 \\
\hline Total & & & & 829,00 & & & & 178816,38 \\
\hline $\begin{array}{l}\text { Prome } \\
\text { dio }\end{array}$ & & & & & 580.75 & & & $215,70 \mathrm{t} / \mathrm{ha}$ \\
\hline
\end{tabular}

(*) De acuerdo a estudios de Aróstegui (1982) y Chambergo (1984), las ramas y bifurcaciones leñosas equivalen al 15 y $25 \%$ del volumen o peso total del fuste del árbol. Considerando la característica de los árboles y arbustos andinos, con troncos retorcidos, bifurcados y copa ramificada; en la vegetación estudiada se incorporó $30 \%$ al volumen determinado del fuste principal. 